\title{
Effective optical response of silicon to sunlight in the finite-difference time-domain method
}

\author{
Alexei Deinega* and Sajeev John \\ Department of Physics, University of Toronto, 60 St. George Street, Toronto, Ontario, Canada, M5S 1A7 \\ ${ }^{*}$ Corresponding author: deinega@physics.utoronto.ca
}

Received September 2, 2011; revised November 3, 2011; accepted November 4, 2011;

posted November 4, 2011 (Doc. ID 153778); published December 26, 2011

\begin{abstract}
The frequency dependent dielectric permittivity of dispersive materials is commonly modeled as a rational polynomial based on multiple Debye, Drude, or Lorentz terms in the finite-difference time-domain (FDTD) method. We identify a simple effective model in which dielectric polarization depends both on the electric field and its first time derivative. This enables nearly exact FDTD simulation of light propagation and absorption in silicon in the spectral range of 300-1000 nm. Numerical precision of our model is demonstrated for Mie scattering from a silicon sphere and solar absorption in a silicon nanowire photonic crystal. @ 2011 Optical Society of America

OCIS codes: $\quad 000.3860,000.4430,350.6050$.
\end{abstract}

The finite-difference time-domain (FDTD) method [1] is widely used in computational electrodynamics for light scattering from arbitrary shaped objects [1], transmission and reflection at various incident angles for planar layers of scatterers [1,2], and photonic band structure of infinite periodic structures $[3,4]$.

Unlike frequency domain methods, the dielectric permittivity $\varepsilon(\omega)$ of dispersive materials in tabular form cannot be directly substituted into the FDTD scheme. Instead, it can be approximated using multiple Debye, Drude, Lorentz [ $\underline{1}]$, or critical point [ $\underline{5}$ ] terms of the form

$$
\begin{gathered}
\varepsilon(\omega)=\varepsilon_{\infty}+\sum_{p=1}^{P} \varepsilon_{p}(\omega), \\
\varepsilon_{p}(\omega)=\frac{a_{p, 0}+a_{p, 1}(-i \omega)}{b_{p, 0}+b_{p, 1}(-i \omega)-b_{p, 2} \omega^{2}},
\end{gathered}
$$

where $\varepsilon_{\infty}$ is permitivitty at infinite frequency, and $a_{p, j}, b_{p, j}$ are real fitting coefficients that do not necessarily have a physical meaning. There are recent reports on successful application of the critical point model (with $a_{p, 1} \neq 0$ ) for the description of the dielectric function of gold [ $\underline{6}, \underline{7}]$, silver $[7,8]$, aluminum, and chromium [7] in the wide wavelength range. This model was implemented in FDTD with the help of the recursive convolution (RC) technique [9].

Dispersion profiles of complex materials cannot always be fitted using a small number, $P$, of terms in (1). Alternately, one can divide the required wavelength range into subranges for separate fittings in each subrange. In this case, multiple FDTD simulations should be performed, followed by merging of the separate results. This makes simulation cumbersome and inefficient.

In traditional fitting, each dielectric susceptibility term (2) consists of either a single imaginary pole (Debye model), two complex poles (Lorentz model), or an imaginary pole plus pole at zero (Drude model) in the complex $\omega$ plane. More flexible fitting (some of which is captured in the critical point model [5]), is enabled by additional terms proportional to $(-i \omega)^{n}$ in both numerators and denominators of (2). In the numerator, they describe direct polarization of the medium by time derivatives of the ap- plied electric field. In the denominator, they correspond to higher order electronic damping ( $n$ odd) or corrections to the electronic dispersion relation in the medium ( $n$ even). Remarkably we find that two $(P=2)$ simple susceptibilities of the form

$$
\varepsilon_{p}(\omega)=\frac{\Delta \varepsilon\left(\omega_{p}^{2}-i \gamma_{p}^{\prime} \omega\right)}{\omega_{p}^{2}-2 i \omega \gamma_{p}-\omega^{2}}
$$

are sufficient to model the entire response of bulk crystalline silicon to sunlight over the wavelength range from 300 to $1000 \mathrm{~nm}$, whereas even a large number of Debye, Lorentz, or Drude terms are inadequate to cover the solar spectrum. In particular, we fit silicon permittivity by two terms of the type (3) with $\left(\omega_{p}, \gamma_{p}\right.$, and $\gamma_{p}^{\prime}$ are in $1 / \mu \mathrm{m}$, and the speed of light is unity) $\varepsilon_{\infty}=1, \Delta \varepsilon_{1}=8.93, \Delta \varepsilon_{2}=1.855$, $\omega_{1}=3.42$ (corresponding to wavelength $\lambda_{1} \approx 0.292 \mu \mathrm{m}$ ), $\omega_{2}=2.72 \quad\left(\lambda_{2} \approx 0.368 \mu \mathrm{m}\right), \quad \gamma_{p 1}=0.425, \quad \gamma_{p 2}=0.123$, $\gamma_{p 1}^{\prime}=0.087$, and $\gamma_{p 2}^{\prime}=2.678$. We find excellent agreement (Fig. 1) between our fit and experimental data [10]. This effective optical response model is of particular value in silicon solar cell FDTD simulations. Previous fitting for silicon by three Lorentz terms (see [11] on silicon textured antireflective coatings) was accurate only for the visible spectrum, and no single fitting with Lorentz terms was found for both visible and near ultraviolet ranges.

While various FDTD methodologies exist for dispersive materials $[1,12,13]$, we implement our effective model using the simple and efficient auxiliary differential equation (ADE) technique [1]].
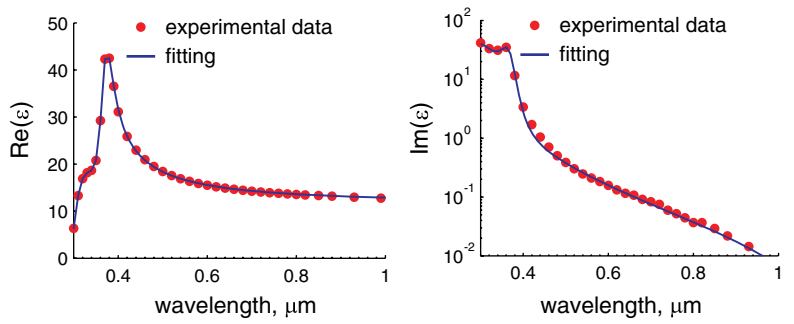

Fig. 1. (Color online) Real and imaginary components of the silicon dielectric permittivity: comparison of the experimental data (dots) with fitting by two terms of the type (3) (curve). 
The effective Ampere's law in time domain for a material described by (1)-(3) is given by

$$
\nabla \times \vec{H}=\varepsilon_{\infty} \frac{d}{d t} \vec{E}+\sigma \vec{E}+\sum_{p} \vec{J}_{p},
$$

where $\vec{H}$ and $\vec{E}$ are the electric and magnetic fields, respectively, $\sigma$ is the material conductivity, and $\vec{J}_{p_{\overrightarrow{ }}}$ is the polarization current response to the electric field $\vec{E}$ associated with $\varepsilon_{p}$ :

$$
\vec{J}_{p}=-i \omega \varepsilon_{p}(\omega) \vec{E} .
$$

For our effective optical response (3), this relation becomes

$$
\vec{J}_{p}(\omega)=-i \omega \frac{\Delta \varepsilon\left(\omega_{p}^{2}-i \gamma_{p}^{\prime} \omega\right)}{\omega_{p}^{2}-2 i \omega \gamma_{p}-\omega^{2}} \vec{E}(\omega) .
$$

Rewriting (6) as

$$
\left(\omega_{p}^{2}-2 i \omega \gamma_{p}-\omega^{2}\right) \vec{J}_{p}(\omega)=\Delta \varepsilon\left(\omega_{p}^{2}-i \gamma_{p}^{\prime} \omega\right) \cdot(-i \omega) \vec{E}(\omega)
$$

and making inverse Fourier transformation, we obtain

$$
\omega_{p}^{2} \vec{J}_{p}+2 \frac{d}{d t} \gamma_{p} \vec{J}_{p}+\frac{d^{2}}{d t^{2}} \vec{J}_{p}=\Delta \varepsilon \omega_{p}^{2} \frac{d}{d t} \vec{E}+\Delta \varepsilon \gamma_{p}^{\prime} \frac{d^{2}}{d t^{2}} \vec{E} .
$$

Finite-difference expression for (8) centered at time step $n$ yields

$$
\begin{aligned}
& \omega_{p}^{2} \vec{J}_{p}^{n}+2 \gamma_{p} \frac{\vec{J}_{p}^{n+1}-\vec{J}_{p}^{n-1}}{2 \Delta t}+\frac{\vec{J}_{p}^{n+1}-2 \vec{J}_{p}^{n}+\vec{J}_{p}^{n-1}}{(\Delta t)^{2}} \\
& \quad=\Delta \varepsilon_{p} \omega_{p}^{2} \frac{\vec{E}^{n+1}-\vec{E}^{n-1}}{2 \Delta t}+\Delta \varepsilon_{p} \gamma_{p}^{\prime} \frac{\vec{E}^{n+1}-2 \vec{E}^{n}+\vec{E}^{n-1}}{(\Delta t)^{2}} .
\end{aligned}
$$

Expressing explicitly $\vec{J}_{p}^{n+1}$ from (9), we get

$$
\vec{J}_{p}^{n+1}=\alpha_{p} \vec{J}_{p}^{n}+\xi_{p} \vec{J}_{p}^{n-1}+\frac{\zeta_{p}^{+} \vec{E}^{n+1}+\zeta_{p}^{-} \vec{E}^{n-1}+\zeta_{p} \vec{E}^{n}}{\Delta t},
$$

where

$$
\begin{gathered}
\alpha_{p}=\frac{2-\omega_{p}^{2}(\Delta t)^{2}}{\gamma_{p} \Delta t+1}, \quad \xi_{p}=\frac{\gamma_{p} \Delta t-1}{\gamma_{p} \Delta t+1}, \\
\zeta_{p}^{ \pm}=\frac{\Delta \varepsilon_{p} \Delta t\left( \pm \omega_{p}^{2} \Delta t / 2+\gamma_{p}^{\prime}\right)}{\gamma_{p} \Delta t+1}, \quad \zeta_{p}=-\frac{2 \Delta \varepsilon_{p} \gamma_{p}^{\prime} \Delta t}{\gamma_{p} \Delta t+1} .
\end{gathered}
$$

The time-stepping scheme for $\left.\vec{J}_{p} \_10\right)$ is applied synchronously with the scheme for $E$ derived from finite- difference discretization of Ampere's law (4) centered at time step $n+1 / 2$ :

$$
\nabla \times \vec{H}^{n+1 / 2}=\varepsilon_{\infty} \frac{\vec{E}^{n+1}-\vec{E}^{n}}{\Delta t}+\sigma \frac{\vec{E}^{n+1}+\vec{E}^{n}}{2}+\sum_{p} \vec{J}_{p}^{n+1 / 2},
$$

where $\vec{J}_{p}^{n+1 / 2}$ is obtained from $(\underline{10})$

$$
\begin{aligned}
\vec{J}_{p}^{n+1 / 2}= & \frac{1}{2}\left(\vec{J}_{p}^{n}+\vec{J}_{p}^{n+1}\right) \\
= & \frac{1+\alpha_{p}}{2} \vec{J}_{p}^{n}+\frac{\xi_{p}}{2} \vec{J}_{p}^{n-1} \\
& +\frac{\zeta_{p}^{+} \vec{E}^{n+1}+\zeta_{p}^{-} \vec{E}^{n-1}+\zeta_{p} \vec{E}^{n}}{2 \Delta t} .
\end{aligned}
$$

Combining (13) and (14), we obtain an explicit timestepping relation for $\vec{E}$ :

$$
\begin{aligned}
\vec{E}^{n+1}= & C_{1} \vec{E}^{n-1}+C_{2} \vec{E}^{n} \\
& +C_{3}\left\{\nabla \times \vec{H}^{n+1 / 2}-\frac{1}{2} \sum_{p}\left[\left(1+\alpha_{p}\right) \vec{J}_{p}^{n}+\xi_{p} \vec{J}_{p}^{n-1}\right]\right\},
\end{aligned}
$$

where

$$
C_{1}=\frac{-\sum_{p} \zeta_{p}^{-}}{2 \varepsilon_{\infty}+\sigma \Delta t+\sum_{p} \zeta_{p}^{+}}
$$

$$
\begin{gathered}
C_{2}=\frac{2 \varepsilon_{\infty}-\sigma \Delta t-\sum_{p} \zeta_{p}}{2 \varepsilon_{\infty}+\sigma \Delta t+\sum_{p} \zeta_{p}^{+}}, \\
C_{3}=\frac{2 \Delta t}{2 \varepsilon_{\infty}+\sigma \Delta t+\sum_{p} \zeta_{p}^{+}} .
\end{gathered}
$$

The resulting $\mathrm{ADE}$ algorithm then consists of three steps. Starting with known values of $\vec{E}^{n-1}, \vec{E}^{n}, \vec{J}_{p}^{n-1}, \vec{J}_{p}^{n}$, and $\vec{H}_{p}^{n+1 / 2}$, we first update $\vec{E}^{n+1}$ using (15). Second, we update $\vec{J}_{p}^{n+1}$ using (10) and just computed $\vec{E}^{n+1}$. Finally, $\vec{H}_{p}^{n+3 / 2}$ is updated from $\vec{E}^{n+1}$ and $\vec{H}_{p}^{n+1 / 2}$ using the standard Yee's discretization for Faradey's law [1].

Another formulation of ADE $[14,15]$, which relates $\vec{E}$ and $\vec{D}$ instead of introducing polarization currents $\vec{J}_{p}$, can likewise be implemented in our model. The ADE technique can be easily extended for the multiple Pade approximants of $\varepsilon(\omega)$ by including the finite-difference discretization of higher order time derivatives.

Our effective optical response model is implemented as a part of the electromagnetic template library (EMTL) [16]. We test this model for Mie scattering from a silicon sphere and solar absorption in silicon nanowires. Geometry of the first numerical test is shown at the inset of Fig. 2. 


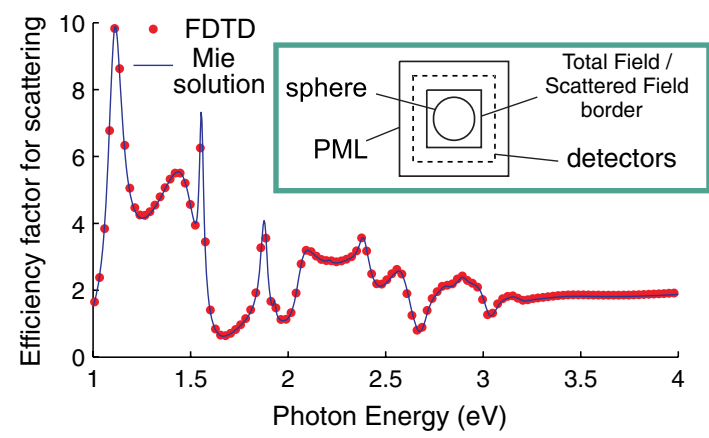

Fig. 2. (Color online) Comparison of scattering $Q_{\text {sca }}$ of a silicon sphere with radius $R=150 \mathrm{~nm}$ for FDTD and exact Mie solution. Inset: scheme of FDTD simulation geometry.

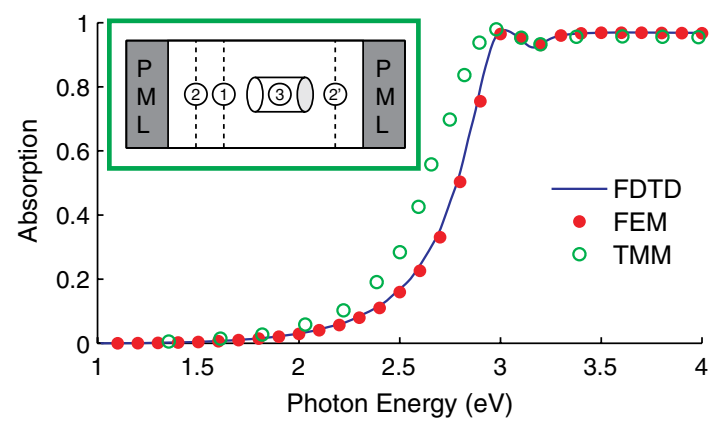

Fig. 3. (Color online) Comparison of absorption of silicon nanowires packed in square lattice (lattice period $a=$ $100 \mathrm{~nm}$. The nanowire's diameter is $d=50 \mathrm{~nm}$ and length is $L=2.33 \mu \mathrm{m}$ ) for FDTD, the finite element method, and the transfer matrix method. Inset: scheme of the FDTD simulation geometry; 1 -generating (TF/SF) border; 2, 2' - detector arrays for reflected and transmitted signals; 3 -periodic cell of nanowires array.

Calculated space (mesh step $\Delta r=7.5 \mathrm{~nm}$ ) is surrounded by absorbing perfectly matched layers (PML) [1]. To reduce undesired numerical reflection from the PML, we use the additional back absorbing layers technique [17]. The total field/scattered field method [1] is used to generate a test plane wave impulse impinging a silicon sphere of radius $R=150 \mathrm{~nm}$. To reduce the error caused by the staircasing effect on rectangular FDTD mesh, subpixel smoothing for dielectric permittivity is used at the sphere borders [18]. The scattered field is measured by detectors that form a closed surface surrounding sphere. Scattering cross section $S$ is calculated as the Poynting vector flux over this surface normalized by the incident flux. Figure 2 depicts the calculated scattering efficiency factor define $\bar{d}$ as the scattering cross section normalized by the sphere geometrical cross section area $Q_{\text {sca }}=S /\left(\pi R^{2}\right)$. We find an excellent agreement between FDTD results and the exact analytical Mie solution.

In the second test, we calculate the absorption spectrum of a silicon nanowire photonic crystal (square lattice) with parameters from [19]: the lattice period $a=100 \mathrm{~nm}$ and the nanowires diameter $d=50 \mathrm{~nm}$ and length $L=2.33 \mu \mathrm{m}$. Here the nanowire crystal is completely suspended in air, and light is incident along the axis of wires with linear polarization.

We use the standard FDTD scheme (see the inset of Fig. 3), where a plane wave impulse is directed onto the structure and transmitted (reflected) fields are recorded, transformed to the frequency domain, and normalized to the incident spectrum, to calculate transmittance $T$ (reflectance $R$ ). Absorption is calculated as $1-T-R$.

Figure 3 depicts the absorption spectra comparison for (a) our FDTD method (mesh step $\Delta r=5 \mathrm{~nm}$ ), (b) the formulation of the finite element method (FEM) adapted to the problem of diffraction by a nanowire array and operating in the frequency domain [20], and (c) transfer matrix method (TMM) results (mesh step $\Delta r=4.5 \mathrm{~nm}$ ) taken from [19]. There is a good agreement between FDTD and TMM results, except for absorption in the $2.2-3 \mathrm{eV}$ range. FDTD results do not change significantly for the decreasing mesh step. The TMM results from [19] may improve if calculated with higher mesh resolution. Excellent agreement over the whole spectral range between FDTD and FEM confirms the accuracy of our scheme.

In summary, we have demonstrated the efficiency of a simple effective optical response model, suitable for FDTD, to simulate silicon throughout near infrared to near ultraviolet range.

We thank Dr. Guillaume Demesy (University of Toronto) for providing us with FEM results on absorption of silicon nanowires. This work is supported in part by the United States Department of Energy contract DEFG02-10ER46754 and the Natural Sciences and Engineering Research Council of Canada.

\section{References}

1. A. Taflove and S. H. Hagness, Computational Electrodynamics: The Finite Difference Time-Domain Method (Artech House, 2005).

2. I. Valuev, A. Deinega, and S. Belousov, Opt. Lett. 33, 1491 (2008).

3. Y. Hao and R. Mittra, FDTD Modeling of Metamaterials: Theory and Applications (Artech House, 2009).

4. A. Deinega, S. Belousov, and I. Valuev, Opt. Lett. 34, 860 (2009).

5. J. Leng, J. Opsal, H. Chu, M. Senko, and D. E. Aspnes, Thin Solid Films 313-314, 132 (1998).

6. P. G. Etchegoin, E. C. Le Ru, and M. Meyer, J. Chem. Phys. 125, 164705 (2006).

7. A. Vial, T. Laroche, M. Dridi, and L. Le Cunff, Appl. Phys. A 103, 849 (2011).

8. J. Lu and Y. Chang, Superlattices Microstruct. 47, 60 (2010).

9. A. Vial, J. Opt. A 9, 745 (2007).

10. M. A. Green and M. Keevers, Progr. Photovol. 3, 189 (1995).

11. A. Deinega, I. Valuev, B. Potapkin, and Yu. Lozovik, J. Opt. Soc. Am. A 28, 770 (2011).

12. J. A. Pereda, A. Vegas, and A. Prieto, IEEE Trans. Microwave Theory Tech. 50, 1689 (2002).

13. S. Liu, N. Yuan, and J. Mo, IEEE Microw. Wireless Compon. Lett. 13, 187 (2003).

14. T. O. Korner and W. Fichtner, Opt. Lett. 22, 1586 (1997).

15. L. J. Prokopeva, J. D. Borneman, and A. V. Kildishev, IEEE Trans. Magn. 47, 1150 (2011).

16. Electromagnetic Template Library, http://kintechlab.com.

17. A. Deinega and I. Valuev, Comput. Phys. Commun. 182, 149 (2011).

18. A. Deinega and I. Valuev, Opt. Lett. 32, 3429 (2007).

19. L. Hu and G. Chen, Nano Lett. 7, 3249 (2007).

20. G. Demesy, F. Zolla, A. Nicolet, and M. Commandre, J. Opt. Soc. Am. A 27, 878 (2010). 